\title{
Addendum to 'Asymptotic inference for a linear stochastic differential equation with time delay'
}

\author{
ALEXANDER A. GUSHCHIN ${ }^{1}$ and UWE KÜCHLER ${ }^{2}$ \\ ${ }^{1}$ Steklov Mathematical Institute, Gubkina 8, 117966 Moscow GSP-1, Russia. \\ E-mail: gushchin@mi.ras.ru \\ ${ }^{2}$ Institut für Mathematik, Humboldt-Universität zu Berlin, Unter den Linden 6, D-10099 Berlin, \\ Germany.E-mail:kuechler@mathematik.hu-berlin.de
}

We strengthen the convergence result proving the local asymptotic mixed normality property in one of the 11 cases considered in our previous paper.

Keywords: local asymptotic mixed normality; maximum likelihood estimator; stochastic differential equations; time delay

In Gushchin and Küchler (1999) we considered the stochastic differential equation

$$
\mathrm{d} X(t)=a X(t) \mathrm{d} t+b X(t-1) \mathrm{d} t+\mathrm{d} W(t), \quad t \geqslant 0,
$$

with initial condition $X(t)=X_{0}(t), t \in[-1,0]$, where $\left(X_{0}(t), t \in[-1,0]\right)$ is a given continuous stochastic process independent of a standard Wiener process $(W(t), t \geqslant 0)$. The parameter $\vartheta=(a, b)^{*}$ is unknown and supposed to be estimated based on $(X(t), t \leqslant T)$. (The sign ${ }^{*}$ denotes matrix or vector transposition.) The solutions $(X(t), t \in[-1, T])$ of the differential equation (1) generate a family $\left(P_{T}^{\vartheta}, \vartheta \in \mathbb{R}^{2}\right)$ of distributions on $\mathbb{C}([-1, T])$. We studied local asymptotic properties of this family and corresponding properties of the maximum likelihood estimators of $\vartheta$ for $T \rightarrow \infty$.

In particular, for a fixed $\vartheta_{0}=(a, b)^{*} \in \mathbb{R}^{2}$ the limit behaviour of

$$
\frac{\mathrm{d} P_{T}^{\vartheta}}{\mathrm{d} P_{T}^{\vartheta_{0}}}(X), \vartheta \in \mathbb{R}^{2},
$$

was studied. It turned out that this behaviour depends strongly on the position of $\vartheta_{0}$, and one has to consider 11 different cases (see Gushchin and Küchler 1999, Table 1, p. 1064). The family $\left(P_{T}^{\vartheta}\right)$ is locally asymptotically normal in one of these cases, it is locally asymptotically mixed normal (LAMN) in another three cases, and it is only locally asymptotically quadratic in five of the remaining cases. As for the last cases, called P1 and P2, we showed that the family $\left(P_{T}^{\vartheta}\right)$ is still locally asymptotically quadratic but has an additional property that we called periodic local asymptotic mixed normality (PLAMN).

Assume that we are in case P2, i.e. $a<1$ and $b<\xi / \sin \xi$, where $\xi$ is the unique number in $(0, \pi)$ with $\xi=a \tan \xi(\xi=\pi / 2$ if $a=0)$, or $a \geqslant 1$ and $b<-\mathrm{e}^{a-1}$. We refer to 
Gushchin and Küchler (1999) for the definition of the random vector $V_{T}$, the random matrix $I_{T}$, and the numbers $v_{0}>0, \xi_{0}>0, A_{0}$ and $B_{0}$. It was shown that the following properties hold (Gushchin and Küchler 1999, Proposition 2.6):

(i)

$$
\log \frac{\mathrm{d} P_{T}^{\vartheta_{0}+\mathrm{e}^{-v_{0}} T_{\mu}}}{\mathrm{d} P_{T}^{\vartheta_{0}}}(X)=\mu^{*} V_{T}-\frac{1}{2} \mu^{*} I_{T} \mu, \quad \mu \in \mathbb{R}^{2} ;
$$

(ii) for $T_{n}=u+n \Delta$, where $u \in[0, \Delta)$ is fixed, $\Delta=\pi / \xi_{0}, n \geqslant 0$,

$$
\left(V_{T_{n}}, I_{T_{n}}\right) \stackrel{d}{\rightarrow}\left(V_{\infty}(u), I_{\infty}(u)\right), \quad n \rightarrow \infty,
$$

where $\left(V_{\infty}(u), I_{\infty}(u)\right) \stackrel{d}{=}\left(I_{\infty}^{1 / 2}(u) Z, I_{\infty}(u)\right)$ and $Z$ is a random vector independent of $I_{\infty}(u)$ and distributed as $\mathscr{N}\left(0, \mathscr{T}_{2}\right)$. The matrix $I_{\infty}(u)$ is given by

$$
I_{\infty}(u)=\left(\begin{array}{cc}
\int_{0}^{\infty} \mathrm{e}^{-2 v_{0} t} U_{0}^{2}(u-t) \mathrm{d} t & \int_{0}^{\infty} \mathrm{e}^{-2 v_{0} t} U_{0}(u-t) U_{2}(u-t) \mathrm{d} t \\
\int_{0}^{\infty} \mathrm{e}^{-2 v_{0} t} U_{0}(u-t) U_{2}(u-t) \mathrm{d} t & \int_{0}^{\infty} \mathrm{e}^{-2 v_{0} t} U_{2}^{2}(u-t) \mathrm{d} t
\end{array}\right),
$$

where

$$
U_{i}(t)=X_{0}(0) \phi_{i}(t)+b \int_{-1}^{0} \phi_{i}(t-s-1) \mathrm{e}^{-v_{0}(s+1)} X_{0}(s) \mathrm{d} s+\int_{0}^{\infty} \phi_{i}(t-s) \mathrm{e}^{-v_{0} s} \mathrm{~d} W(s),
$$

in which

$$
\phi_{i}(t)=A_{i} \cos \left(\xi_{0} t\right)+B_{i} \sin \left(\xi_{0} t\right), \quad i=0,2
$$

and

$$
\left(\begin{array}{l}
A_{2} \\
B_{2}
\end{array}\right)=\mathrm{e}^{-v_{0}}\left(\begin{array}{cc}
\cos \xi_{0} & -\sin \xi_{0} \\
\sin \xi_{0} & \cos \xi_{0}
\end{array}\right)\left(\begin{array}{l}
A_{0} \\
B_{0}
\end{array}\right)
$$

We have referred to this asymptotic behaviour as PLAMN to indicate that the cluster points of $\left(V_{T}, I_{T}\right)$ have the same structure as in the LAMN case but that $\left(V_{T}, I_{T}\right)$ converges in distribution only if $T$ runs to infinity through a grid in such a way that the fractional part of $T / \Delta$ tends to a limit.

The purpose of this note is to show that in case P2 one can prove the usual LAMN property. To this end, we look for matrices $\Phi_{T}, T \geqslant 0$, such that

$$
\Phi_{T}^{*}\left(\begin{array}{c}
U_{0}(T-t) \\
U_{2}(T-t)
\end{array}\right)
$$

does not depend on $T$. In view of (4) and (5), this will be the case if

$$
\Phi_{T}^{*}\left(\begin{array}{cc}
A_{0} & A_{2} \\
B_{0} & B_{2}
\end{array}\right)\left(\begin{array}{c}
\cos (T-t) \\
\sin (T-t)
\end{array}\right)
$$


is independent of $T$. Using (6), after elementary calculations one obtains that the latter property holds if and only if

$$
\Phi_{T}=\Phi_{0}\left(\begin{array}{cc}
\cos \left(\xi_{0} T\right)-\sin \left(\xi_{0} T\right) \cot \xi_{0} & -\mathrm{e}^{-v_{0}} \sin \left(\xi_{0} T\right) \csc \xi_{0} \\
\mathrm{e}^{v_{0}} \sin \left(\xi_{0} T\right) \csc \xi_{0} & \cos \left(\xi_{0} T\right)+\sin \left(\xi_{0} T\right) \cot \xi_{0}
\end{array}\right)
$$

Define $\Phi_{T}$ according to (7) with $\Phi_{0}:=\mathscr{T}_{2}$, where $\mathscr{T}_{2}$ is the identity matrix. Then

$$
\begin{aligned}
\left(\begin{array}{c}
\tilde{U}_{0}(t) \\
\tilde{U}_{2}(t)
\end{array}\right):= & \Phi_{T}^{*}\left(\begin{array}{c}
U_{0}(T-t) \\
U_{2}(T-t)
\end{array}\right)=X_{0}(0)\left(\begin{array}{c}
A_{0} \cos \left(\xi_{0} t\right)-B_{0} \sin \left(\xi_{0} t\right) \\
\mathrm{e}^{-v_{0}}\left(A_{0} \cos \left(\xi_{0}(t+1)\right)-B_{0} \sin \left(\xi_{0}(t+1)\right)\right)
\end{array}\right) \\
& +b \int_{-1}^{0}\left(\begin{array}{c}
A_{0} \cos \left(\xi_{0}(t+s+1)\right)-B_{0} \sin \left(\xi_{0}(t+s+1)\right) \\
\mathrm{e}^{-v_{0}}\left(A_{0} \cos \left(\xi_{0}(t+s+2)\right)-B_{0} \sin \left(\xi_{0}(t+s+2)\right)\right)
\end{array}\right) \mathrm{e}^{-v_{0}(s+1) X_{0}(s) \mathrm{d} s} \\
& +\int_{0}^{\infty}\left(\begin{array}{c}
A_{0} \cos \left(\xi_{0}(t+s)\right)-B_{0} \sin \left(\xi_{0}(t+s)\right) \\
\mathrm{e}^{-v_{0}}\left(A_{0} \cos \left(\xi_{0}(t+s+1)\right)-B_{0} \sin \left(\xi_{0}(t+s+1)\right)\right)
\end{array}\right) \mathrm{e}^{-v_{0} s} \mathrm{~d} W(s) .
\end{aligned}
$$

Extend the definition of $I_{\infty}(u)$ to $u \in \mathbb{R}_{+}$by the same formula (3). Then

$$
\Phi_{T}^{*} I_{\infty}(T) \Phi_{T}=\int_{0}^{\infty} \mathrm{e}^{-2 v_{0} t} \Phi_{T}^{*}\left(\begin{array}{c}
U_{0}(T-t) \\
U_{2}(T-t)
\end{array}\right)\left(\begin{array}{c}
U_{0}(T-t) \\
U_{2}(T-t)
\end{array}\right)^{*} \Phi_{T} \mathrm{~d} t,
$$

and, therefore, the matrix $\tilde{I}_{\infty}:=\Phi_{T}^{*} I_{\infty}(T) \Phi_{T}$ does not depend on $T$ and is given by

$$
\tilde{I}_{\infty}=\left(\begin{array}{cc}
\int_{0}^{\infty} \mathrm{e}^{-2 v_{0} t} \tilde{U}_{0}^{2}(t) \mathrm{d} t & \int_{0}^{\infty} \mathrm{e}^{-2 v_{0} t} \tilde{U}_{0}(t) \tilde{U}_{2}(t) \mathrm{d} t \\
\int_{0}^{\infty} \mathrm{e}^{-2 v_{0} t} \tilde{U}_{0}(t) \tilde{U}_{2}(t) \mathrm{d} t & \int_{0}^{\infty} \mathrm{e}^{-2 v_{0} t} \tilde{U}_{2}^{2}(t) \mathrm{d} t
\end{array}\right)
$$

Put

$$
\tilde{V}_{T}:=\Phi_{T}^{*} V_{T}, \quad \tilde{I}_{T}:=\Phi_{T}^{*} I_{T} \Phi_{T}
$$

Proposition 1. In case $\mathrm{P} 2$ the family $\left(P^{\vartheta}, \vartheta \in \mathbb{R}^{2}\right)$ is LAMN at every $\vartheta_{0}$ :

$$
\log \frac{\mathrm{d} P_{T}^{\vartheta_{0}+\mathrm{e}^{-v_{0} T} \Phi_{T} \mu}}{\mathrm{d} P_{T}^{\vartheta_{0}}}(X)=\mu^{*} \tilde{V}_{T}-\frac{1}{2} \mu^{*} \tilde{I}_{T} \mu
$$

and

$$
\left(\tilde{V}_{T}, \tilde{I}_{T}\right) \stackrel{d}{\rightarrow}\left(\tilde{V}_{\infty}, \tilde{I}_{\infty}\right)
$$

where $\left(\tilde{V}_{\infty}, \tilde{I}_{\infty}\right) \stackrel{d}{=}\left(\tilde{I}_{\infty}^{1 / 2} Z, \tilde{I}_{\infty}\right)$ and $Z$ is a vector independent of $\tilde{I}_{\infty}$ and distributed as $\mathscr{N}\left(0, \mathscr{T}_{2}\right)$.

Proof. Equation (8) is obvious from (2). 
It was shown in the proof of Proposition 2.6 that

$$
I_{T}-I_{\infty}(T) \stackrel{P}{\rightarrow} 0 .
$$

Thus

$$
\tilde{I}_{T} \stackrel{P}{\rightarrow} \tilde{I}_{\infty}
$$

because the matrices $\Phi_{T}$ are uniformly bounded. Now the joint convergence (9) follows from the stable limit theorem for martingales as in the proofs of Propositions 2.2-2.4 in Gushchin and Küchler (1999).

Remark. Contrary to the case just considered, in case P1 one cannot avoid periodic behaviour of the likelihood and prove the LAMN property by choosing an appropriate matrix norming. For a general statement of this type for delay processes, see Putschke (2000).

\section{Acknowledgements}

The authors express their thanks to anonymous referees, whose comments led to a more detailed explanation of how to construct the matrices $\Phi_{T}$.

\section{References}

Gushchin, A.A. and Küchler, U. (1999) Asymptotic inference for a linear stochastic differential equation with time delay. Bernoulli, 5, 1059-1098.

Putschke, U. (2000) Affine stochastische Funktionaldifferentialgleichungen und lokal asymptotische Eigenschaften ihrer Parameterschätzungen. Doctoral dissertation, Humboldt-Universität zu Berlin.

Received June 2000 and revised March 2001 